\title{
Bond-Selective Excitation and Following Displacement of Ge Atoms in GeTe/ $\mathrm{Sb}_{2} \mathrm{Te}_{3}$ Superlattice
}

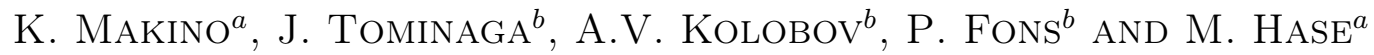 \\ ${ }^{a}$ Institute of Applied Physics, University of Tsukuba, 1-1-1 Tennodai, Tsukuba 305-8573, Japan \\ ${ }^{b}$ Functional Nano-phase-change Research Team, Nanodevice Innovation Research Center \\ National Institute of Advanced Industrial Science and Technology \\ Tsukuba Central 4, 1-1-1 Higashi, Tsukuba 305-8562, Japan
}

\begin{abstract}
Phase change random access memory devices made from chalcogenides compounds, such as $\mathrm{Ge}_{2} \mathrm{Sb}_{2} \mathrm{Te}_{5}$, have attracted much attention because of their high-speed read-write and low power consumption capabilities. The phase change in $\mathrm{Ge}_{2} \mathrm{Sb}_{2} \mathrm{Te}_{5}$ is thought to be characterized by the displacement of Ge atoms, accompanying relaxation of surrounding $\mathrm{Sb}$ and Te atoms. Here we examine a new approach, that is the manipulation of $\mathrm{Ge}-\mathrm{Te}$ bonds using linearly-polarized femtosecond near-infrared optical pulses. As a result, $p$-polarized pump pulses are found to be more effective in inducing the precursor of phase change, probably due to the atomic arrangements along the unique axis of the superlattice structure.
\end{abstract}

PACS: 78.47.J-, 63.22.Np, 63.20.kd, 63.20.kk

\section{Introduction}

Pseudobinary alloys along the $\mathrm{GeTe} / \mathrm{Sb}_{2} \mathrm{Te}_{3}$ pseudobinary tie-line have been used in a wide range of phase-change memory applications including DVD, Blu-ray disk, and electrical memory [1]. $\mathrm{Ge}_{2} \mathrm{Sb}_{2} \mathrm{Te}_{5}$ (GST) is often considered to be a prototypical phase change memory compound and has been used in both commercial electrical and optical phase-change devices. Phase-change materials demonstrate pronounced differences in optical and electronic properties upon transformation between the disordered and crystalline phases; the transformation can be induced by heating with pico- or nanosecond optical or electrical pulses. The underlying mechanism of the phase change process has been examined both theoretically and experimentally. In the transformation from the disordered to crystalline phase, creation of the long-range order of the crystalline state leads to the formation of a delocalized-bonding state, often referred to as resonant bonding [2] in contrast to the more typical covalent bonding of the disordered state [3]. The change in bonding is accompanied by correspondingly large changes in optical and electrical properties. It has been shown that long-range order can be manipulated by taking advantage of the bonding energy hierarchy of the crystalline state $[4,5]$. In addition, recent studies have demonstrated unusual ultrafast response from photoexcited carriers and coherent local vibrations [6, 7]. Furthermore, the excitation of an anisotropic carrier distribution by linearly polarized pulses in solids has been shown to induce bond-selective excitation in Ge [8].
Besides the elucidation of phase change mechanisms for alloy materials described above, new artificial structures based upon $\mathrm{Ge}-\mathrm{Sb}$ - Te phase-change alloys have recently been developed to further enhance switching properties. Chong et al. reported that phase-change electrical memory based upon superlattice (SL)-like (GeTe)/ $\left(\mathrm{Sb}_{2} \mathrm{Te}_{3}\right)$ provided better performance with respect to the switching speed and programing current as well as durability [9]. Subsequently, crystallographically strongly-oriented GST-SL with superlattice wavelengths only a few monolayers thick were developed [10] based on the phase-change model of Kolobov and workers [11] and were shown to greatly reduce the energy requirements for switching due to a reduction in dimensionality. Furthermore, some of the current authors demonstrated ultrafast phase change from the disordered to crystalline phases within 1 ps by manipulation of the local vibrational modes originating from local $\mathrm{GeTe}_{4}$ units in GST-SL [7]. This result implies that the phase change in GST-SL is characterized by the displacement of Ge atoms without a nucleation process. For further development in both operation time and energy consumption of the data storage technology, it is important to utilize coherent control techniques in atomically-designed materials. However, the detailed mechanism of the phase change in GST is still under debate and further investigations are required.

In this study, we examine the possibility of bond-selective excitation for Ge atoms in disordered GST-SL by tuning the polarization of the pump pulse and observing the resulting changes in the ultrafast local vi- 
brational motion of the $\mathrm{A}_{1}$ mode of $\mathrm{GeTe}_{4}$ employing coherent phonon spectroscopy (CPS). In crystallographical oriented material such as the SL, a particular orientation of a linearly polarized pulse would be expected to induce an anisotropic photoexcited carrier distribution as well as a subsequent anisotropic electron-phonon and hole-phonon coupling [8, 12, 13]. Here we demonstrate the harnessing of coherent phonon generation to induce a driving force for the selective displacement of $\mathrm{Ge}$ atoms from tetrahedral-like to octahedral-like coordination through anisotropic electron-phonon coupling by application of a linearly polarized pulse. CPS is a powerful experimental method to study the ultrafast dynamics of structural change on the sub-picosecond time scale, which is applied to semimetals [14], ferromelectric materials $[15,16]$, and the Mott insulators [17, 18]. Previously reported results for CPS in GST revealed that a Ge-related phonon mode is affected by the phase change $[19,20]$. In GST-SL, the frequency of the $\mathrm{A}_{1}$ mode of tetrahedral-like coordinated $\mathrm{Ge}$ in the disordered phase is $3.84 \mathrm{THz}$. On the other hand, that of octahedral-like coordinated $\mathrm{Ge}$ in the crystalline state is at $3.68 \mathrm{THz}$. The origin of the modification of the phonon mode in GST-SL can be attributed to the displacement of Ge atoms between tetrahedral-like (disordered state) and octahedral-like coordination (crystalline state). The phase change can thus be distinguished by the direct observation of the phonon frequency.

\section{Experimental}

Figure 1 illustrates the schematic view of our experimental setup. In our crystallization model based on earlier experimental and theoretical results [7, 10, 11], a Ge atom coordinated by four Te atoms is displaced to a vacancy site leading to the formation of three bonds at the vacancy side and the breaking of one bond on the opposite side. In that sense, irradiation of GST-SL with an optical pulse linearly polarized along the [111] direction would selectively excite a weaker bond [8], because the weaker bond in GST-SL is aligned along the [111] direction. In our sample, the [111] direction corresponds to the sample normal and $p$-polarized light can excite the weaker bond preferentially.

The sample investigated was $\left[\mathrm{GeTe} / \mathrm{Sb}_{2} \mathrm{Te}_{3}\right]_{20}$ consists of alternatively deposited $5 \mathrm{~nm}$ thick GeTe sublayers and $5 \mathrm{~nm}$ thick $\mathrm{Sb}_{2} \mathrm{Te}_{3}$ sublayers, which has been described in detail elsewhere [10]. Time-resolved pump-probe reflectivity measurements were performed utilizing a near-infrared optical pulse with 20 fs duration with a wavelength of $850 \mathrm{~nm}$ generated by a Ti:sapphire laser oscillator. The linearly polarized pump and probe pulses were focused onto the sample surface by a $50 \mathrm{~mm}$ focal length focusing mirror. The pump fluence at the sample surface was $50 \mu \mathrm{J} / \mathrm{cm}^{2}$. Coherent local vibrations were generated by the pump pulse over a range of incident and polarization angles. The transient reflectivity change measured by the probe pulse $(\Delta R / R)$ was

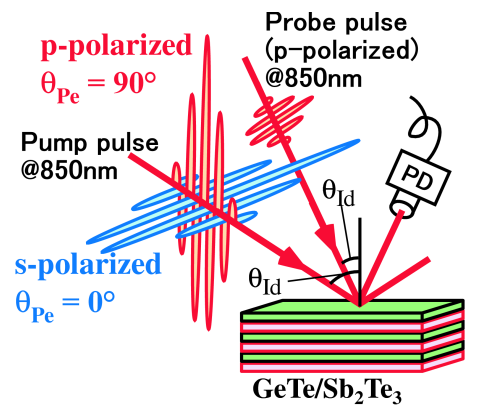

Fig. 1. Schematic view of selective bond excitation using a linearly-polarized pulse. A linearly polarized pump pulse with a polarization angle between pure $s$ -polarization and $p$-polarization introduces local vibrational motion of $\mathrm{GeTe}_{4}$ subunits. The polarization angle of the pump pulse $\left(\theta_{\mathrm{Pe}}\right)$ was varied $180^{\circ}$ and the change in reflectivity was recorded as a function of pump-probe time delay by photodiode (PD). The incident angles of the pump $\left(\theta_{\mathrm{Ie}}\right)$ and probe pulses $\left(\theta_{\mathrm{Id}}\right)$ were $\approx 35^{\circ}$ and $\approx 30^{\circ}$ from the surface normal, respectively.

recorded as a function of pump-probe time delay $(\tau)$ at room temperature. Pump power was much lower than the threshold for thermal crystallization and the increase in temperature was negligible. The polarization angle of pump pulse $\left(\theta_{\mathrm{Pe}}\right)$ was varied between $0^{\circ}(s$-polarization $)$ to $180^{\circ}$ ( $s$-polarization) via $90^{\circ}$ ( $p$-polarization), while that of probe pulse $\left(\theta_{\mathrm{Pd}}\right)$ was fixed at $90^{\circ}$.

\section{Results and discussions}

Figure 2 shows the transient reflectivity change $(\Delta R / R)$ for three different $\theta_{\mathrm{Pe}}$ as a function of $\tau$ for a GST-SL. At $\tau=0$, coherent local vibrations appear superimposed on the electronic response due to the generation of photoexcited carriers. Figure $2 \mathrm{~b}$ shows the Fourier transformed (FT) spectra obtained from Fig. 2a, in which mainly the local $\mathrm{A}_{1}$ mode of $\mathrm{GeTe}_{4}$ is observed [20]. The frequency of the local $\mathrm{A}_{1}$ mode of $\mathrm{GeTe}_{4}$ is slightly higher than the previously reported values $(3.84 \mathrm{THz})$, which can be reasonably accounted for by the difference of the incident angle of the pump pulses because phonon frequency is a function of wave vector. By increasing $\theta_{\mathrm{Pe}}$ from $0^{\circ}$ to $90^{\circ}$, a red-shift of the $\mathrm{A}_{1}$ mode of $\mathrm{GeTe}_{4}$ as well as enhancement of the intensity was observed.

Figure 3 shows the pump polarization angle dependence of the FT intensity and the frequency of the coherent local $A_{1}$ mode of $\mathrm{GeTe}_{4}$. It is found that as $\theta_{\mathrm{Pe}}$ approaches $90^{\circ}$, an increase in FT intensity and a decrease in phonon frequency were clearly observed. These results would suggest that excitation with a $p$-polarization pulse enables not only enhancement of the oscillation amplitude of the local $\mathrm{GeTe}_{4}$ mode, but also affects the rearrangement of $\mathrm{Ge}$ atoms from tetrahedral-like $\mathrm{GeTe}_{4}$ coordination toward octahedral-like $\mathrm{GeTe}_{6}$ coordination.

In order to gain a deeper understanding of the polarization dependence on the local $\mathrm{A}_{1}$ mode of $\mathrm{GeTe}_{4}$, 

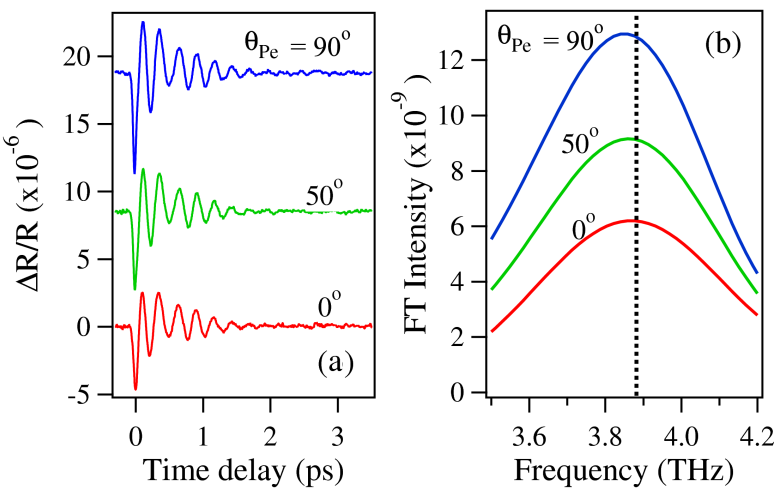

Fig. 2. (a) Time-resolved $\Delta R / R$ signals measured with $\theta_{\mathrm{Pe}}=0^{\circ}, 50^{\circ}, 90^{\circ}$. (b) FT spectra obtained from (a). $\quad \theta_{\mathrm{Pe}}=0^{\circ}$ corresponds to $s$-polarization, $\theta_{\mathrm{Pe}}=90^{\circ}$ corresponds to $p$-polarization. $\theta_{\mathrm{Pd}}$ was fixed at $90^{\circ}$ ( $p$-polarized). Dashed line indicates the center frequency $(3.88 \mathrm{THz})$ of the peak measured with $\theta_{\mathrm{Pe}}=0^{\circ}$.
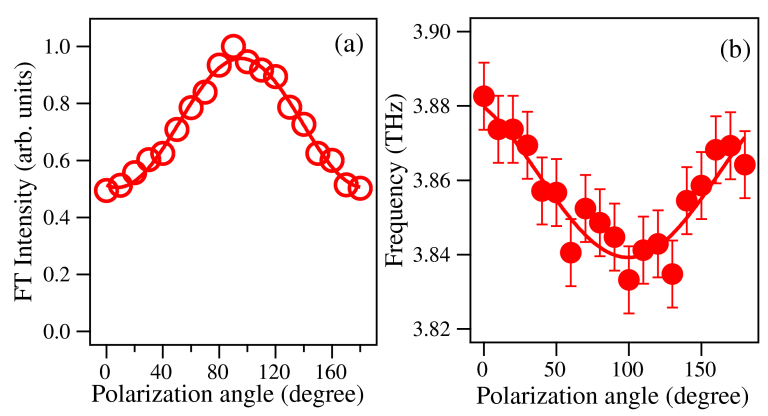

Fig. 3. Polarization dependence of (a) the FT intensity and (b) the frequency of the coherent local $\mathrm{A}_{1}$ mode of $\mathrm{GeTe}_{4}$ measured with $0 \leqq \theta_{\mathrm{Pe}} \leqq 180^{\circ}$ and $\theta_{\mathrm{Pd}}=90^{\circ}$.

the generation mechanism of the coherent local vibration should be considered. Under the aforementioned experimental conditions, the coherent local mode would be expected to be induced by resonant impulsive stimulated Raman scattering (ISRS) [21]. A plausible scenario for the polarization dependence of $\mathrm{A}_{1}$ mode of $\mathrm{GeTe}_{4}$ is as follows. The $p$-polarized pulse excitation would generate anisotropic carrier distribution along the [111] axis, in which Ge-Te bonds are aligned, then the $s$-polarized pulse excitation. Then, due to the anisotropic carrierphonon coupling along Ge-Te line, Ge atoms displace from tetrahedral site into octahedral site as a precursor of the phase change, which is observed as the phonon softening. This displacement thought to be introduced within 100 fs which was confirmed by a wavelet analysis (not shown).

The hypothesis about the bond-selective excitation in GST-SL can be also supported by the following comparison with the observed pump fluence dependence. When we increased the pump fluence, only $\mathrm{a} \approx 0.04 \mathrm{THz}$ red-shift in the frequency of the local $\mathrm{GeTe}_{4}$ mode was ob- served, together with a eighteenfold increase in the FT intensity of the $\mathrm{GeTe}_{4}$ in a range of $47-284 \mu \mathrm{J} / \mathrm{cm}^{2}$ [7]. On the other hand, in the present study, the bond-selective excitation process is found to yield the same amount of frequency shift $(\approx 0.04 \mathrm{THz}$; see Fig. $3 \mathrm{~b})$ with a much smaller enhancement of the FT intensity (twofold). The comparison described above clearly demonstrates that bond-selective excitation with $p$-polarized light is more effective for phase-change modification via the local $\mathrm{GeTe}_{4}$ vibration.

\section{Conclusion}

We have demonstrated bond-selective excitation in GST-SL. Ultrafast CPS has been carried out with various pump-pulse polarization. When the polarization of pump pulse was purely $p$-polarization, not only an increase in the phonon intensity but also the decrease in the phonon frequency were observed, over the sufficiently lower pump fluence range. The result implies the displacement of Ge atoms (and surrounding $\mathrm{Sb}$ and $\mathrm{Te}$ atoms) along [111] direction due to the anisotropic selective excitation of the weaker bond with the $p$-polarized pulse.

\section{Acknowledgments}

This work was supported in part by KAKENHI-22340076 from MEXT, Japan, Grant-in-Aid for JSPS Fellows from JSPS, Japan and "Innovation Research Project on Nanoelectronics Materials and Structures Research and development of superlatticed chalcogenide phase-change memory based on new functional structures" from METI, Japan.

\section{References}

[1] M. Wuttig, N. Yamada, Nat. Mat. 6, 824 (2007).

[2] L. Pauling, Nature of Chemical Bond, Cornell Univ. Press, New York 1939.

[3] K. Shportko, S. Kremers, M. Woda, D. Lencer, J. Robertson, M. Wuttig, Nat. Mat. 7, 653 (2008).

[4] A.V. Kolobov, M. Krbal, P. Fons, J. Tominaga, T. Uruga, Nat. Chem. 3, 311 (2011).

[5] P. Fons, H. Osawa, A.V. Kolobov, T. Fukaya, M. Suzuki, T. Uruga, N. Kawamura, H. Tanida, J. Tominaga, Phys. Rev. B 82, 041203 (2010).

[6] M. Konishi, H. Santo, Y. Hongo, K. Tajima, M. Hosoi, T. Saiki, Appl. Opt. 49, 3470 (2010).

[7] K. Makino, J. Tominaga, M. Hase, Opt. Exp. 19, 1260 (2011).

[8] T. Pfeifer, W. Kütt, H. Kurz, R. Schölz, Phys. Rev. Lett. 69, 3248 (1992).

[9] T.C. Chong, L.P. Shi, R. Zhao, P.K. Tan, J.M. Li, K. Lee, X.S. Miao, A.Y. Du, C.H. Tung, Appl. Phys. Lett. 88, 122114 (2006).

[10] J. Tominaga, P. Fons, A.V. Kolobov, T. Shima, T.C. Chong, R. Zhao, H.K. Lee, L.P. Shi, Jpn. J. Appl. Phys. 47, 5763 (2008). 
[11] A.V. Kolobov, P. Fons, A.I. Frenkel, A.L. Ankudinov, J. Tominaga, T. Uruga, Nat. Mat. 3, 703 (2004).

[12] T.P. Devereaux, T. Cuk, Z.-X. Shen, N. Nagaosa, Phys. Rev. Lett. 93, 117004 (2004).

[13] F. Carbone, D.-S. Yang, E. Giannini, A.H. Zewail, Proc. Natl. Acad. Sci. 105, 20161 (2008).

[14] M. Hase, M. Kitajima, S.I. Nakashima, K. Mizoguchi, Phys. Rev. Lett. 88, 067401 (2002).

[15] M. Hase, M. Kitajima, S. Nakashima, K. Mizoguchi, Appl. Phys. Lett. 83, 4921 (2003).

[16] R. Lu, M. Hase, M. Kitajima, S. Nakashima, S. Sugai, Phys. Rev. B 75, 012107 (2007).

[17] A. Cavalleri, T. Dekorsy, H.H.W. Chong, J.C. Kieffer, R.W. Schoenlein, Phys. Rev. B 70, 161102(R) (2004).
[18] C. Kubler, H. Ehrke, R. Huber, R. Lopez, A. Halabica, R.F. Haglund, Jr., A. Leitenstorfer, Phys. Rev. Lett. 99, 116401 (2007).

[19] M. Först, T. Dekorsy, C. Trappe, M. Laurenzis, H. Kurz, B. Bechevet, Appl. Phys. Lett. 77, 1964 (2000).

[20] M. Hase, Y. Miyamoto, J. Tominaga, Phys. Rev. B 79, 174112 (2009).

[21] G.A. Garrett, T.F. Albrecht, J.F. Whitaker, R. Merlin, Phys. Rev. Lett. 77, 3661 (1996). 